\title{
Human Urine-derived Stem Cells Improve Bladder Function After Partial Bladder Outlet Obstruction: Preliminary Data and the microRNA-mRNA Expression Profile of Bladder Tissue
}

\section{Menjiang Tu}

Shenzhen University General Hospital

\section{Rui Wang}

Shenzhen University General Hospital

Pei Zhu

Shenzhen University General Hospital

\section{Qingqing Wang}

Third Military Medical University Second Affiliated Hospital: Xinqiao Hospital

\section{Bishao Sun}

Third Military Medical University Second Affiliated Hospital: Xinqiao Hospital

Keshi Lu

Shenzhen University General Hospital

Jiawei Zhang

Shenzhen University General Hospital

Weijie Xie

Shenzhen University General Hospital

Huan Guo

Shenzhen University General Hospital

Shulin Li

Shenzhen University General Hospital

\section{Yuqi Wu}

Shenzhen University General Hospital

Xiangwei Wang ( $\square$ winn0324@szu.edu.cn )

Dept. of Urology, Shenzhen University General Hospital, Shenzhen, China.

\section{Research}

Keywords: partial bladder outlet obstruction, human urine-derived stem cells, gene expression profiles, microRNA 
Posted Date: June 16th, 2021

DOl: https://doi.org/10.21203/rs.3.rs-614750/v1

License: (c) (i) This work is licensed under a Creative Commons Attribution 4.0 International License. Read Full License 


\section{Abstract}

Background: Partial bladder outlet obstruction (pBOO), a common urological disease, often results in bladder tissue inflammation and tissue remodeling. Human urine-derived stem cells (hUSCs) have shown therapeutic benefits in various organ injury models. We used a rat model to investigate the effect of hUSCs on bladder function in pBOO rats and explore the miRNA and gene expression profiles in bladder tissue using RNA-sequencing.

Methods: In total, 18 rats were randomly and evenly assigned to three groups: a sham surgery group, a pBOO without USCs therapy group, and a pBOO with USCs therapy group (treated with hUSCs six times every other week). All rats were subjected to routine urodynamic monitoring. Detrusor muscle strips were evaluated and pathophysiology was assessed. Finally, altered miRNA and mRNA expression profiles of bladder tissue were examined using RNA-sequencing and bioinformatic analysis technology.

Results: After USC treatment, urodynamic monitoring revealed elevated bladder compliance and maximal voiding pressure, declined end filling pressure and voided volume, and improved detrusor contractility and carbachol sensitivity in pBOO rats. Histology and TUNEL assay showed reduced collagen deposition and muscle cell apoptosis in bladder tissue. The differential expression of eight miRNAs in pBOO rats was reversed by USC treatment. Bioinformatic analysis identified miR-142 and miR-9a as the two largest nodes of differentially expressed miRNAs in the miRNA-gene interaction network. The Kyoto Encyclopedia of Genes and Genomes analysis revealed enrichment of multiple significant pathways, including those involved in necroptosis and cytokine-cytokine receptor interactions.

Conclusions: This is the first study to reveal the protective effect of hUSCs on bladder function and bladder remodeling in pBOO rats. The miRNA and mRNA expression levels differed in the bladder of pBOO rats with and without USC treatment. Although the mechanism underlying these effects have not been fully elucidated, necroptosis and cytokine-cytokine receptor interaction related pathways may be involved.

\section{Background}

Partial bladder outlet obstruction (pBO0) is a common urological disorder in which a partial obstruction of any part between the bladder outlet and external urethra is observed. The most common causes of pBOO are benign prostate hyperplasia in adult men, posterior urethral valves in newborns, pelvic prolapse in women, and urethral stricture caused by iatrogenic injury. In patients with moderate or severe long-term pBOO, an increase in bladder outlet resistance pressure and intravesical pressure results in bladder tissue inflammation, detrusor hypertrophy, connective tissue accumulation in the bladder wall, and tissue remodeling and decreased bladder compliance [1-4]. Even after the obstruction is relieved, bladder dysfunction remains. Symptoms such as waiting for urine, weak urination, frequent urination, and nocturia may be maintained or even worsen. 
Because of their self-renewal ability, multidirectional differentiation, and proliferation, stem cells have demonstrated tissue repair capabilities during the process of tissue remodeling and fibrosis in multiple organ systems. Studies have confirmed that mesenchymal stem cells can inhibit the inflammatory response and fibrosis process of bladder tissue after pBO0, thereby improving bladder function [5]. Urine-derived stem cells (USCs) are pluripotent stem cells with multidirectional differentiation potential. USCs are easier to extract directly from urine than mesenchymal stem cells, which is more convenient and economical and does not require invasive surgery. For patients without kidney disease, USCs can be extracted directly from the patient's urine, which has minor ethical issues and lower risk of autoimmune reactions.

In this study, we used human USCs (hUSCs) in pBOO rats, aiming to clarify the effect of USCs on bladder function by observing bladder urodynamics and histological changes, and investigated the microRNA (miRNA) and mRNA expression profiles of bladder smooth muscle tissue using high-throughput sequencing. We used bioinformatics methods to clarify the potential molecular pathways of USCs acting on bladder tissue.

\section{Methods}

\section{Ethics statement}

The experiments were approved by the Animal Care and Use Committee of Shenzhen University General Hospital and complied with the Guide for the Care and Use of Laboratory Animals, Eighth Edition (2011). Written informed consent was obtained from all the donors prior to donating urine samples.

\section{Animals and study design}

The eighteen adult male Sprague-Dawley rats used in this study were purchased from the Center for Experimental Animals of Guangzhou University of Chinese Medicine. All rats weighed $190-210 \mathrm{~g}$ and were housed at $20-26^{\circ} \mathrm{C}$ under a standard $12 \mathrm{~h} / 12 \mathrm{~h}$ light-dark cycle. The rats were randomly and evenly assigned to three groups: a sham surgery group (control group), a pBOO without USC therapy group (pBOO group), and pBOO with USC therapy group (pBOO+USCs group).

\section{Induction of pBOO models}

The twelve rats in the $\mathrm{pBOO}$ and $\mathrm{pBOO+USCs}$ groups underwent urethral ligation under isoflurane anesthesia to establish the pBOO model, as described previously [4]. Briefly, with the rat in the supine position, a midline suprapubic incision was made, and the bladder neck and urethra were exposed. A 1.0$\mathrm{mm}$ inner diameter catheter was placed around the proximal urethra. A 4-0 silk ligature was tied around the urethra and tube. After checking the tightness, the tube was removed, and the incision was closed. Six rats in the control group only underwent an operation to expose the bladder without urethral ligation.

\section{Isolation and culture of USCs}


USCs were collected from the urine of five healthy male donors aged 19-25 years. The collection, isolation, and identification methods were described in our previous study [6]. Briefly, urine samples were centrifuged at $500 \times g$ for $5 \mathrm{~min}$ to collect the cells. The cell pellet was gently resuspended in a mixed medium consisting of embryo fibroblast medium and keratinocyte serum-free medium (1:1 ratio) and seeded in 24-well plates (passage $(P)$ ). Individual hUSCs appeared 3-5 days after the initial seeding and reached $70 \%-80 \%$ confluency after another 3-4 days. The cells were trypsinized and transferred to 6-well plates (P1). Finally, the cells were transferred to a 100-mm culture dish (P2) for expansion. P3 hUSCs were used in the experiments.

\section{Treatment with USCs}

The pBOO model was successfully established by urodynamic analysis seven weeks after the urethral ligation procedure. Hence, all rats in the $\mathrm{pBOO+USCs}$ group were administered a tail vein injection of USCs $\left(2 \times 10^{6}\right.$ cells suspended in $0.2 \mathrm{~mL}$ phosphate-buffered saline [PBS] ) six times every other week. Rats in $\mathrm{pBOO}$ group received an injection of an equal volume of PBS using the same process.

\section{Urodynamics}

Urodynamics studies were performed at the $7^{\text {th }}$ week post-urethral ligation to determine the baseline urodynamic values, and urethral ligation was released for rats in the pBOO groups. All rats were subjected to urodynamics studies $7,11,15$, and 19 weeks after the urethral ligation. Under isoflurane anesthesia through an abdominal incision, a 27-gauge double lumen catheter was inserted into the bladder and connected to a pressure transducer (MLT488, ADinstuments, Australia). The bladder was emptied using a syringe and the abdomen was covered with a warm gauze. Saline was infused through the catheter, at a constant rate of $0.3 \mathrm{~mL} / \mathrm{min}$. The following parameters were recorded: end-filling pressure, voided volume, maximal voiding pressure, bladder capacity, and residual volume. Bladder volume change $(\Delta \mathrm{V})$ was calculated as the perfusion volume from infusion to urine leakage, and bladder pressure change $(\Delta \mathrm{P})$ was calculated as the bladder leak point pressure minus pressure before bladder perfusion. Bladder compliance (compliance, $\mathrm{C}$ ) was calculated using the formula: $\Delta V / \Delta \mathrm{P}$.

\section{Measurement of bladder muscle strip tension}

At the end of the experiments, all rats were euthanized using an isoflurane anesthetic overdose. The bladder was rapidly removed and fixed in Krebs-Henseleit solution at $4{ }^{\circ} \mathrm{C}$. The mucosa was carefully removed under a microscope and excised into longitudinal muscle strips (8 $\mathrm{mm} 2 \mathrm{~mm}$ ) using a doubleedged knife. All muscle strips were transferred to a 10-mL organ bath filled with Krebs-Henseleit solution. The bath was maintained at $37{ }^{\circ} \mathrm{C}$ under an atmosphere of $95 \% \mathrm{O}_{2}$ and $5 \% \mathrm{CO}_{2}$. After warming for $30 \mathrm{~min}$ without tension, one end of each strip was ligated to a force transducer (MLT488, ADinstuments, Australia), and the other end was fixed to the bottom of the fixing plate with 4-0 silk ligature. The force transducer-linked tension sensor was connected to the computer to detect and record the signal with a Powerlab multichannel biological signal analysis system (MI224, ADinstuments, Australia). The finetuning spiral was adjusted to slowly pull the muscle strip to increase the tension of the muscle strip. 
Contraction was quantified by measuring the force generated in millinewton $(\mathrm{mM})(\mathrm{g}=9.81)$. The basic value of tension was $0.7 \mathrm{mN}$, which was maintained for $15 \mathrm{~min}$. After the muscle strips were balanced, $10^{-4} \mathrm{M}$ carbachol was added to stimulate the muscle strips, and the maximum contraction value of the muscle strips was recorded.

\section{Histological examination}

Half of the bladder was snap frozen in liquid nitrogen for microarray expression profiling and bioinformatics analysis. The remaining tissues were fixed in $4 \%$ paraformaldehyde and embedded in paraffin for staining. The sections were stained with hematoxylin-eosin $(\mathrm{HE})$ to observe the general morphology of the bladders and Masson's trichrome to evaluate the level of tissue fibrosis. All sections were evaluated under a light microscope (Olympus, Tokyo, Japan).

\section{TUNEL assay}

A one-step TUNEL apoptosis assay kit (Beyotime Biotechnology, Shanghai, China) was used to evaluate apoptosis levels in the bladder smooth muscle. Briefly, the sections were regularly hydrated and immersed in citric acid buffer $(0.01 \mathrm{mM}, \mathrm{pH}=6)$, followed by incubation with $10 \%$ bovine serum albumin for $30 \mathrm{~min}$. Consequently, the sections were reacted with TUNEL reaction solution for $20 \mathrm{~min}$ and then with DAPI for $10 \mathrm{~min}$ in a dark chamber. Finally, after regular dehydration and transparency, the sections were observed under a fluorescence microscope (Olympus, Tokyo, Japan) and analyzed by a pathologist in a blinded manner.

\section{RNA sequencing}

At the end of the experiment, total RNA was extracted from a section of the bladder tissue obtained from two rats in the control group, two rats in the pBOO group, and four rats in the pBOO+USCs group, and processed for RNA sequencing. A total of $1 \mu \mathrm{g}$ RNA per sample was used as the input material for the RNA sample preparations. Sequencing libraries were generated using the rRNA-depleted RNA using the NEBNext ${ }^{\circledR}$ Ultra ${ }^{\text {TM }}$ Directional RNA Library Prep Kit for Illumina ${ }^{\circledR}$ (NEB, USA), following the manufacturer's recommendations. Library quality was assessed using an Agilent Bioanalyzer 2100 system (Agilent, USA). Clustering of the index-coded samples was performed on a cBot Cluster Generation System using TruSeq PE Cluster Kit v3-cBot-HS (Illumina), according to the manufacturer's instructions. After cluster generation, the libraries were sequenced on an Illumina Hiseq 3000 platform, and 150-bp paired-end reads were generated. Reference genome and gene model annotation files were downloaded directly from the Genome website. The mapped reads of each sample were assembled using both Scripture (beta2) and Cufflinks (v2.1.1). Raw read counts were then used as input to DESeq to calculate the normalized signal for each transcript in the samples. The $p$ value was adjusted using the $q$ value. A $q$ value $<0.01$ and $\mid \log 2$ (foldchange)| $>1$ were set as the thresholds for significantly differential expression by default. For small RNA sequencing, it was mapped to the reference sequence using Bowtie [7]. miRBase20.0 was used as a reference [8]. The target gene of miRNA was predicted using psRobot_tar in miRanda for 
animals [9]. In the present study, FUNRICH (http://www.funrich.org/) was used to perform Gene Ontology (GO) and Kyoto Encyclopedia of Genes and Genomes (KEGG) pathway analyses.

\section{Statistical analysis}

All values are presented as the means SD. Statistical analyses were performed using the SPSS ver. 22.0 (SPSS Inc., Chicago, IL, USA). The S-N-K analysis of variance was applied to evaluate the differences among the different rat groups. Statistical significance was set at $p<0.05$.

\section{Results}

\section{Urodynamics}

Urodynamic studies in all groups revealed the highest bladder compliance in controls and the lowest compliance in both pBOO groups in the $7^{\text {th }}$ week post-urethral ligation $(48.578 .61 \mathrm{vs} .21 .003 .87 \mu \mathrm{L} / \mathrm{cm}$ $\mathrm{H}_{2} \mathrm{O}$, respectively, $\mathrm{p}<0.01$ ). After two injections of USCs, compliance began to improve in the pBOO+USCs group in the $15^{\text {th }}$ week compared with that in the pBOO group (38.35 2.81 vs. $16.574 .69 \mu \mathrm{L} / \mathrm{cm} \mathrm{H}_{2} \mathrm{O}$, respectively, $p<0.01$ ) and was similar to that of controls in the $19^{\text {th }}$ week (48.50 7.28 vs. 50.078 .04 $\mu \mathrm{L} / \mathrm{cm} \mathrm{H}_{2} \mathrm{O}$, respectively, $\mathrm{p}=0.89$ ) (Fig. $\left.1 \mathrm{~A}\right)$. The maximal voiding pressure in the $\mathrm{pBOO}$ group was significantly lower than that in the control group in the $7^{\text {th }}$ week (24.32 $3.22 \mathrm{vs.} 46.8010 .17 \mathrm{~cm} \mathrm{H}_{2} \mathrm{O}$, respectively, $p<0.01$ ), whereas $p B 00+U S C s$ began to show an increasing pressure from the $15^{\text {th }}$ week (38.31 5.21 vs. $22.612 .60 \mathrm{~cm} \mathrm{H}_{2} \mathrm{O}$, respectively, $\mathrm{p}<0.01$ ) (Fig. 1B). End-filling pressure in $\mathrm{pBOO}$ rats was higher than that in controls (10.96 2.07 vs. $6.841 .80 \mathrm{~cm} \mathrm{H}_{2} \mathrm{O}$, respectively, $p=0.006$ ) and increased from the $7^{\text {th }}$ week to $19^{\text {th }}$ week $\left(10.962 .07\right.$ in $7^{\text {th }}$ week vs. $16.151 .68 \mathrm{~cm} \mathrm{H}_{2} \mathrm{O}$ in $19^{\text {th }}$ week, $\left.\mathrm{p}<0.01\right)$, whereas the pressure in pBOO+USCs rats decreased to the control level (9.03 0.99 vs. $7.261 .94 \mathrm{~cm} \mathrm{H}_{2} \mathrm{O}$, respectively, $\mathrm{p}=0.359$ ) in the $15^{\text {th }}$ week (Fig. $1 \mathrm{C}$ ). All pBOO and pBOO+USCs rats had significantly higher residual volume than control rats $\left(0.700 .11\right.$ vs. $1.360 .19 \mathrm{~mL}$, respectively, in the $7^{\text {th }}$ week $\left.[p<0.01]\right)$;

however, USCs considerably suppressed the upward trend from the $15^{\text {th }}$ week $(1.260 .12 \mathrm{vs} .1 .930 .10 \mathrm{~mL}$, $\mathrm{p}<0.01$ ) (Fig. 1D). Moreover, pBOO rats had lower voided volume than control rats in the $7^{\text {th }}$ week (238.77 27.11 vs. $337.8421 .49 \mu \mathrm{L}, \mathrm{p}<0.01$ ), but the pBOO+USCs group showed an increasing trend and finally had similar voided volume as the control group in the $19^{\text {th }}$ week (340.75 $16.93 \mathrm{vs}$. $316.5116 .23 \mu \mathrm{L}$, respectively, $p=0.119$ ) (Fig. 1E). In addition, a significant increase in the bladder compacity was observed in all pBOO rats compared with that in the control (1.54 0.14 vs. $1.040 .14 \mathrm{~mL}$, respectively, $\mathrm{p}<$ 0.01). Although USCs reversed the trend in $\mathrm{PBOO+USCs}$ group from the $15^{\text {th }}$ week (1.59 $0.23 \mathrm{vs}$. 2.170 .17 $\mathrm{mL}, \mathrm{p}<0.01$ ), the bladder compacity was not decreased to the level of the control (1.52 $0.27 \mathrm{vs} .1 .080 .16$ $\mathrm{mL}$, respectively, $\mathrm{p}<0.01$ ) (Fig. 1F).

\section{Histology and TUNEL assay}


HE staining showed that the bladder wall thickness was higher, the urothelium was flatter, and structural damage of detrusor smooth muscle was worse in the pBOO group than in the control group, but treatment with USCs significantly alleviated these histological changes (Fig. 2A). Masson's trichrome staining revealed increased collagen, disorderly and loosely arranged detrusor muscle bundles, and an obviously widened gap between muscle bundles in the pBOO group compared with the control group and the pBOO+USCs group (Fig. 2B). There was a marked increase in the number of TUNEL-positive cells in the $\mathrm{pBOO}$ group compared with the control group, whereas USC treatment significantly decreased the number of apoptotic cells in the bladder (Fig. 2C).

\section{Assessment of detrusor contractility}

In the detrusor tension tests, the bladder detrusor contraction amplitudes of pBOO rats were significantly lower than those of control rats. Carbochol elevated the contraction amplitudes at different concentrations $\left(10^{-6}\right.$ to $\left.10^{-4} \mathrm{M}\right)$ in the three groups, but the bladder detrusor of pBOO rats had the lowest sensitivity to carbochol. The effect of carbochol on the amplitudes of contraction in pBOO+USCs rats was significantly higher than that in $\mathrm{pBOO}$ rats (4.58 0.89 vs. $2.850 .23 \mathrm{mM}$, respectively, $p<0.01)$ but lower than that in control rats (4.58 0.89 vs. $6.040 .26 \mathrm{mM}$, respectively, $p<0.01$ ) (Fig. 2D).

\section{Bioinformatic analysis of miRNA-mRNA}

Bioinformatic analysis revealed differentially expressed genes (DEGs) and differentially expressed miRNAs (DEMs) in the bladder wall tissue among the control, pBOO, and pBOO+USCs groups in rats. Below, we provide results for miRNAs and mRNAs that were regulated in the pBOO and USCs treated relative to controls. The comparisons are $\mathrm{pBOO}$ relative to the control group and $\mathrm{pBOO+USCs}$ relative to the pBOO group.

The volcano plots in Figure $3 a$ and $b$ show DEMs in rat bladder wall tissues of the control, pBOO, and pBOO+USCs groups. The distribution of all miRNAs with respect to significance ( $y$-axis) vs. fold change (x-axis) is shown in the volcano plot. Compared with the control group, the present study identified 62 DEMs (40 upregulated and 22 downregulated) and 1686 DEGs (981 upregulated and 705 downregulated) in the pBOO group. Compared with the pBOO group, we identified 42 DEMs (25 upregulated and 17 downregulated) and 757 DEGs (241 upregulated and 516 downregulated) in the pBO0+USCs group. The expression of eight DEMs that induced expression changes in $\mathrm{pBOO}$ rats relative to controls was reversed by USC treatment (Fig. 3).

Five of them, miR-142-3p, miR-142-5p, miR-187-3p, miR-503-5p, and 17_19667_star, were upregulated in pBOO rats (Fig. 3A) and downregulated by USC treatment (Fig. 3B). The Venn diagrams of downregulated mRNAs in rats from pBOO vs. control group (pBOO vs. ctrl mRNA_lo) and upregulated mRNAs in rats from pBOO+USCs vs. pBOO group (pBOO+USCs vs. pBOO mRNA_up) and target genes of the above five DEMs identified three DEGs (Fig. 3). 
Three of the eight DEMs, miR-9a-3p, miR-9a-5p, and 1_1401, were downregulated in pBO0 rats (Fig. 3A) and upregulated by USC treatment (Fig. 3B). Similarly, the Venn diagrams of upregulated mRNAs in rats from $\mathrm{pBOO}$ vs. control group (pBOO vs. ctrl mRNA_up) and downregulated mRNAs in rats from pBOO+USCs vs. pBOO group (pBOO+USCs vs. pBOO mRNA_lo) and target genes of the above three DEMs identified 17 DEGs (Fig. 3). The changes in the levels of these DEMs in the three groups are shown in the gene trend map in Figure $3 \mathrm{C}$ and $\mathrm{D}$.

Following the above analysis, an miRNA-gene interaction network was constructed by integrating the target genes with DEGs. As shown in Figure 4A, the pBOO and USC-associated DEM-DEG interaction network was constructed, which included 23 nodes, 4 of which were DEMs, and 22 connections. miR-9a$3 p$ and miR-9a-5p were the two largest nodes of the four DEMs and interacted with 13 and 5 DEGs, respectively.

GO analysis showed that the most significantly enriched GO terms that corresponded to the above target DEGs were "superoxide-generating NADPH oxidase activity" (ontology: MF), "NADPH oxidase complex" (ontology: $\mathrm{CC}$ ) and "semaphorin-plexin signaling pathway involved in axon guidance" (ontology: BP) (Fig. $4 \mathrm{~B})$.

Furthermore, KEGG analysis revealed that multiple significant pathways were enriched, which were primarily associated with leukocyte transendothelial migration, chemokine signaling pathway, cytokinecytokine receptor interaction, necroptosis, nucleotide-binding and oligomerization domain (NOD)-like receptor signaling pathway, and apoptosis signaling pathway (Fig. 4C).

\section{Discussion}

PBOO initiates a pathophysiological cascade of events in the bladder wall, including inflammation, hypoxia, collagen deposition, smooth muscle hypertrophy, apoptosis, and fibrosis. The obstructed bladder modifies its structure to compensate for the increased resistance to flow, and significant hypoxia ensues owing to the high resistance to flow and consequent high intravesical pressure [1-4]. Studies in 2 weekobstructed rabbit models showed a larger capacity and lower intravesical pressure and compliance of the bladder [2,4]. In addition, studies in Sprague-Dawley rats have shown an increase in end-filling pressure, residual volume, and bladder capacity as well as a decrease in maximal voiding pressure and bladder compliance $[2,10]$. Some parameters vary with the duration of obstruction time, such as voided volume. Yuan et al. revealed that voided volume increases in 3 week-obstructed rats and decreases in 6 weekobstructed group [3]. Bladder strip contractility tension and tension sensitivity decrease in various animal models, including mice, rats, and rabbits $[4,10,11]$.

Currently, effective treatments are lacking to improve bladder function after long-term moderate to severe pBOO in clinical practice. Studies have confirmed the effect of MSCs on the bladder function of pBOO animal models based on the potential of stem cells in tissue reconstruction and organ repair engineering $[5,12-14]$. However, the mechanism has not been explored at the genetic and 
molecular levels. In addition, the disadvantages of MSCs, including the invasive extraction process and high cost, have limited their future clinical applications. Compared with MSCs, USCs are advantageous owing to the simple, noninvasive, and low-cost harvesting methods. Studies have revealed the nephronprotective effect of USCs on renal function via anti-inflammatory, antioxidative stress, and antifibrotic activities in acute and chronic kidney disease [15,16]. Moreover, USC-exosomes showed pro-neurogenesis effects in the rat brain after ischemic stroke as well as exerted pro-angiogenesis effects and improved erectile dysfunction in diabetic rats, thereby protecting their bladder function [17-19]. At present, there are no reports on the effects of USCs on the bladder function in patients with $\mathrm{pBOO}$.

As we aimed to explore the effect of hUSCs on bladder function and morphology in moderate or severe long-term pBOO, we designed a long-term pBOO rat model and dynamically observed the changes from 7to 19-week-obstructed rats. Similar to previous studies, we observed elevated end-filling pressure, residual volume, and bladder capacity and declined maximal voiding pressure, voided volume, and bladder compliance in the pBOO group compared with the control group. In addition, once a low-compliance bladder was formed, cystometric parameters did not improve without intervention, even if the urethral ligature was removed. Some parameters, including end-filling pressure, residual volume, and bladder capacity, consistently increased after ligature removal, which consequently resulted in worsening of bladder function. Similar to the case of most patients with moderate to severe long-term prostatic hyperplasia, the lower urinary tract symptoms of patients with pBOO cannot be relieved even if they have accepted resection of the prostate to relieve bladder outlet obstruction. After treatment with USCs, some cystometric parameters were significantly improved, including elevated bladder compliance and maximal voiding pressure, declined end-filling pressure, and voided volume. Although residual volume and bladder compacity did not recover, there was no further deterioration after USC treatment compared with the pBOO group. The detrusor contractility and carbachol sensitivity were also improved after USC treatment. Results from the histological analysis and TUNEL assay showed that the improvement of these functions was mainly related to the reduction of collagen deposition in the extracellular matrix and the reduction of muscle cell apoptosis.

Furthermore, the urinary bladder was exposed to mechanical stress and treated with USCs, which undoubtedly resulted in modification of gene and protein expression profiles in epithelial and smooth muscle cells, consequently altering the ultrastructure and physiology of cells in the bladder wall. To elucidate the molecular mechanisms underlying this complex process, this study conducted bioinformatic analysis of miRNA-mRNA expression profiles of rats in the three groups. We focused on the numerous miRNAs and mRNAs that were significantly differentially expressed in the bladder following pBOO and but underwent reversed expression following USC treatment. We identified miR-142 and miR-9a as the two largest nodes of DEMs in the miRNA-gene interaction network.

MiR-142-3p and miR-142-5p have been observed to be overexpressed in the bladder tissue of pBOO mice [20]. A previous study confirmed that miR-142-5p is overexpressed in the bladder of patients with pBOO [21]. MiR-142-3p and miR-142-5p influence inflammation and immune response, the levels of which have been shown to decrease in mouse models of cardiac hypertrophy. miR-142-5p targets the

Page $10 / 19$ 
acetyltransferase p300 and is implicated in cardiac growth; additionally, an increase in miR-142 expression during cardiac growth is critical for cell survival [22,23]. The in vivo inhibition of miR-142-5p with locked nucleic acid-modified oligonucleotides reduces CCL4-induced liver fibrosis and bleomycininduced lung fibrosis in mice. Furthermore, macrophages from the tissue samples of patients with liver cirrhosis and idiopathic pulmonary fibrosis display increased levels of miR-142-5p [24]. Therefore, miR142-5p regulates macrophage profibrogenic gene expression during chronic inflammation. In this study, miR-142-3p and miR-142-5p were overexpressed in pBOO rats, whereas their expression was suppressed by USCs, indicating that miR-142 participates in the antifibrosis effect of USCs in pBOO rats.

Studies have revealed that miR-9a participates in anti-inflammatory and anti-apoptotic functions and is related to neurogenesis. The downregulation of miR-9a-5p expression could increase the levels of NLRP1 inflammasome proteins, cleaved caspase-1, interleukin (IL)-1 b, and IL-18. In contrast, the overexpression of miR-9a-5p markedly prevents the abnormal expression of these proteins and is accompanied by the downregulation of NLRP1 expression in ischemia-like conditions, both in vivo and in vitro [25]. MiR-9 prevents cardiac dysfunction and inhibits cardiomyocyte apoptosis in myocardial infarction mouse hearts [26]. However, a study have indicated that miR-9a-5p is a novel regulator of HSCs in the setting of increased pressure and hepatic fibrosis in rats and that the overexpression of miR-9a-5p following hepatic stellate cell activation perpetuates the fibrotic response [27]. In our study, we found that both miR$9 a-3 p$ and miR-9a-3p levels were decreased in pBOO rats and increased in USC-treated pBOO rats. They may protect bladder function by reducing the inflammatory response of bladder tissue and reducing detrusor cell apoptosis, which needs to be confirmed through further studies.

To obtain insights into the classification of miRNAs, we conducted GO and KEGG analyses to predict the target genes and possible pathways. The results revealed various $\mathrm{GO}$ terms associated with superoxidegenerating NADPH oxidase activity, NADPH oxidase complex, semaphorin receptor activity, semaphorinplexin signaling pathway involved in axon guidance, oxidoreductase activity, acting on $N A D(P) H$, oxygen as an acceptor, and hydrogen peroxide biosynthetic process. In addition, it was observed that various pathways were involved, including leukocyte transendothelial migration, chemokine signaling pathway, cytokine-cytokine receptor interaction, necroptosis, NOD-like receptor signaling pathway, and apoptosis. The necroptosis signaling pathway and cytokine-cytokine receptor interaction signaling pathway may be responsible for the pathological alterations observed in this study and provide a strategy for therapeutic intervention, following further investigation. The necroptosis signaling pathway is a programmed form of necrosis. It can be initiated by different stimuli, such as tumor necrosis factor (TNF) and TNF-related apoptosis-inducing ligand. It has previously been demonstrated that cytokine and immune response pathways, transforming growth factor, nitric oxide signaling, and hypertrophic PI3K/AKT signaling pathways are shared among all pBOO phenotypes. AP- 1 and NF-kB are the dominant transcription factors, and TNF-a is the top upstream regulator [21]. However, the classic fibrosis-related pathways, such as the transforming growth factor beta signaling pathway, were not identified in this analysis. Therefore, USCs may inhibit the initiation of the pathophysiological cascade of events in the bladder wall via the necroptosis and cytokine-cytokine receptor interaction signaling pathways. Additional research is 
needed to verify the exact roles of the identified miRNAs, genes, and signaling pathways in the pathogenesis of bladder remodeling induced by pBOO.

\section{Conclusions}

To the best of our knowledge, this study is the first to identify the protective effects of hUSCs on bladder function and bladder remodeling in pBOO rats. Treatment with USCs elevated bladder compliance and maximal voiding pressure, reduced end-filling pressure and voided volume, strengthened detrusor contractility, and increased sensitivity to carbachol in pBOO rats. These alterations were associated with a reduction in collagen deposition in the extracellular matrix and a reduction in cell apoptosis. Alterations in miRNA and mRNA expression levels in the bladder of pBOO rats with and without USCs were observed. Bioinformatics analysis of these alterations may help to better understand the molecular mechanisms underlying the effects of USCs in pBOO rats. miR-142 and miR-9a may play regulatory roles via anti-inflammatory, anti-apoptotic, and antifibrotic effects. Although the mechanisms by which these effects occur have not been fully elucidated, USCs may exert their benefits via necroptosis and cytokine-cytokine receptor interaction signaling pathways. Further studies are needed to verify these initial outcomes on the potential of USC-mediated bladder repair in the setting of obstructive injury.

\section{Abbreviations}

$\mathrm{DEG}=$ differentially expressed gene

DEM = differentially expressed miRNA

$\mathrm{GO}=$ Gene Ontology

$\mathrm{HE}=$ hematoxylin-eosin

KEGG = Kyoto Encyclopedia of Genes and Genomes

pBOO $=$ partial bladder outlet obstruction

PBS $=$ phosphate-buffered saline

TNF = tumor necrosis factor

USCs = urine-derived stem cells

\section{Declarations}

Ethics approval and consent to participate 
The experiments were approved by the Animal Care and Use Committee of Shenzhen University General Hospital and complied with the Guide for the Care and Use of Laboratory Animals, Eighth Edition (2011). Written informed consent was obtained from all the donors prior to donating urine samples.

Consent for publication

Not applicable.

Availability of data and materials

The datasets generated and/or analyzed in the current study are available in the GEP repository (accession\#GSE171367).

Competing interests

The authors declare that they have no competing interests.

Funding

This study was sponsored by the Science and Technology Innovation Commission Foundation of Shenzhen (Grant No. JCYJ20190808141013454 and JCYJ20180305124827261)

\section{Authors' contributions}

MT designed the methods and was a major contributor to writing the manuscript. RW and PZ contributed to the animal model and conducted the animal procedure. QW and BS contributed to the isolation and culture of USCs. KL conducted the urodynamics studies. JZ analyzed and interpreted the bioinformatic data. WX and HG analyzed and interpreted the urodynamic data. SL contributed to the histological examination. YW and XW made substantial contributions to the conception of the study and revised the manuscript. All authors read and approved the final manuscript.

\section{Acknowledgements}

Not applicable.

\section{References}

1. Duan LJ, Cao QF, Xu D, Liu HL, Qi J. Bioinformatic analysis of microRNA-mRNA expression profiles of bladder tissue induced by bladder outlet obstruction in a rat model. Mol Med Rep. 2017;16:4803-10.

2. Malkowicz SB, Wein AJ, Elbadawi A, Van Arsdalen K, Ruggieri MR, Levin RM. Acute biochemical and functional alterations in the partially obstructed rabbit urinary bladder. J Urol. 1986;136;1324-9.

3. Yuan X, Wu S, Lin T, He D, Li X, Liu S, et al. Role of nitric oxide synthase in bladder pathologic remodeling and dysfunction resulting from partial outlet obstruction. Urology. 2011;77:1008.e1-8. 
4. Oka M, Fukui T, Ueda M, Tagaya M, Oyama T, Tanaka M. Suppression of bladder oxidative stress and inflammation by a phytotherapeutic agent in a rat model of partial bladder outlet obstruction. $\mathrm{J}$ Urol. 2009;182:382-90.

5. Wiafe B, Adesida AB, Churchill T, Kadam R, Carleton J, Metcalfe PD. Mesenchymal stem cell therapy inhibited inflammatory and profibrotic pathways induced by partial bladder outlet obstruction and prevented high-pressure urine storage. J Pediatr Urol. 2019;15:254.e1-10.

6. Wang Q, Zhao J, Wu C, Yang Z, Dong X, Liu Q, et al. Large conductance voltage and Ca(2+)-activated $\mathrm{K}(+)$ channels affect the physiological characteristics of human urine-derived stem cells. Am J Transl Res. 2017;9:1876-85.

7. Langmead B, Trapnell C, Pop M, Salzberg SL. Ultrafast and memory-efficient alignment of short DNA sequences to the human genome. Genome Biol. 2009;10:R25.

8. Friedländer MR, Mackowiak SD, Li N, Chen W, Rajewsky N. miRDeep2 accurately identifies known and hundreds of novel microRNA genes in seven animal clades. Nucleic Acids Res. 2012;40:37-52.

9. Enright AJ, John B, Gaul U, Tuschl T, Sander C, Marks DS. MicroRNA targets in Drosophila. Genome Biol. 2003;5:R1.

10. Zeng J, Xie K, Jiang C, Mo J, Lindström S. Bladder mechanoreceptor changes after artificial bladder outlet obstruction in the anesthetized rat. Neurourol Urodyn. 2012;31:178-84.

11. Austin JC, Chacko SK, DiSanto M, Canning DA, Zderic SA. A male murine model of partial bladder outlet obstruction reveals changes in detrusor morphology, contractility and Myosin isoform expression. $J$ Urol. 2004;172:1524-8.

12. Al-Saikan B, Ding J, Tredget E, Metcalfe P. Benefits of mesenchymal stem cells after partial bladder outlet obstruction. Can Urol Assoc J. 2016;10:E1-6.

13. Woo LL, Tanaka ST, Anumanthan G, Pope JC 4th, Thomas JC, Adams MC, et al. Mesenchymal stem cell recruitment and improved bladder function after bladder outlet obstruction: preliminary data. J Urol. 2011;185:1132-8.

14. Wiafe B, Adesida A, Churchill T, Metcalfe P. Mesenchymal stem cells inhibit hypoxia-induced inflammatory and fibrotic pathways in bladder smooth muscle cells. World J Urol. 2018;36:1157-65.

15. Sun B, Luo X, Yang C, Liu P, Yang Y, Dong X, et al. Therapeutic effects of human urine-derived stem cells in a rat model of cisplatin-induced acute kidney injury in vivo and in vitro. Stem Cells Int. 2019;2019:8035076.

16. Zhang C, George SK, Wu R, Thakker PU, Abolbashari M, Kim TH, et al. Reno-protection of urinederived stem cells in a chronic kidney disease rat model induced by renal ischemia and nephrotoxicity. Int 
17. Ouyang B, Xie Y, Zhang C, Deng C, Lv L, Yao J, et al. Extracellular vesicles from human urine-derived stem cells ameliorate erectile dysfunction in a diabetic rat model by delivering proangiogenic microRNA. Sex Med. 2019;7:241-50.

18. Ling X, Zhang G, Xia Y, Zhu Q, Zhang J, Li Q, et al. Exosomes from human urine-derived stem cells enhanced neurogenesis via miR-26a/HDAC6 axis after ischaemic stroke. J Cell Mol Med. 2020;24:640-54.

19. Dong X, Zhang T, Liu Q, Zhu J, Zhao J, Li J, et al. Beneficial effects of urine-derived stem cells on fibrosis and apoptosis of myocardial, glomerular and bladder cells. Mol Cell Endocrinol. 2016;427:21-32.

20. Gheinani AH, Köck I, Vasquez E, Baumgartner U, Bigger-Allen A, Sack BS, et al. Concordant miRNA and mRNA expression profiles in humans and mice with bladder outlet obstruction. Am J Clin Exp Urol. 2018;6:219-33.

21. Gheinani AH, Kiss B, Moltzahn F, Keller I, Bruggmann R, Rehrauer H, et al. Characterization of miRNAregulated networks, hubs of signaling, and biomarkers in obstruction-induced bladder dysfunction. $\mathrm{JCI}$ Insight. 2017;2:e89560.

22. Iguchi N, Hou A, Koul HK, Wilcox DT. Partial bladder outlet obstruction in mice may cause E-cadherin repression through hypoxia induced pathway. J Urol. 2014;192:964-72.

23. Sharma S, Liu J, Wei J, Yuan H, Zhang T, Bishopric NH. Repression of miR-142 by p300 and MAPK is required for survival signalling via gp130 during adaptive hypertrophy. EMBO Mol Med. 2012;4:617-32.

24. Su S, Zhao Q, He C, Huang D, Liu J, Chen F, et al. miR-142-5p and miR-130a-3p are regulated by IL-4 and IL-13 and control profibrogenic macrophage program. Nat Commun. 2015;6:8523.

25. Cao Y, Zhang H, Lu X, Wang J, Zhang X, Sun S, et al. Overexpression of microRNA-9a-5p ameliorates NLRP1 inflammasome-mediated ischemic injury in rats following ischemic stroke. Neuroscience. 2020;444:106-17.

26. Yang D, Yu J, Liu HB, Yan XQ, Hu J, Yu Y, et al. The long non-coding RNA TUG1-miR-9a-5p axis contributes to ischemic injuries by promoting cardiomyocyte apoptosis via targeting KLF5. Cell Death Dis. 2019;10:908.

27. Qi F, Hu JF, Liu BH, Wu CQ, Yu HY, Yao DK, et al. MiR-9a-5p regulates proliferation and migration of hepatic stellate cells under pressure through inhibition of Sirt1. World J Gastroenterol. 2015;21:9900-15.

\section{Figures}




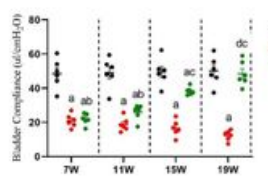

A

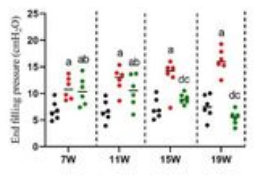

c

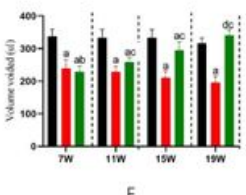

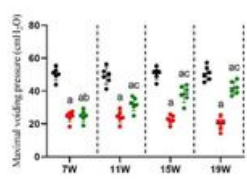

B

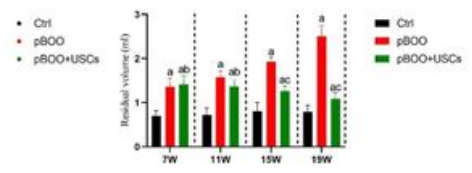

D

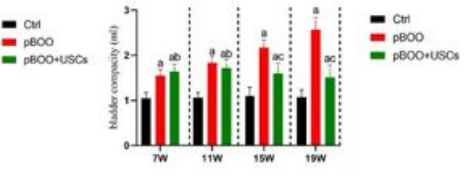

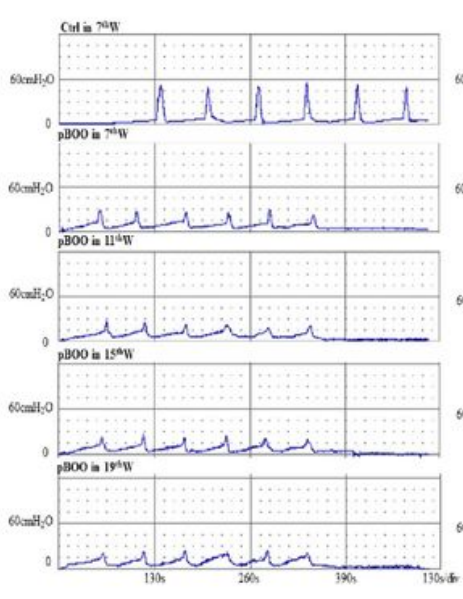

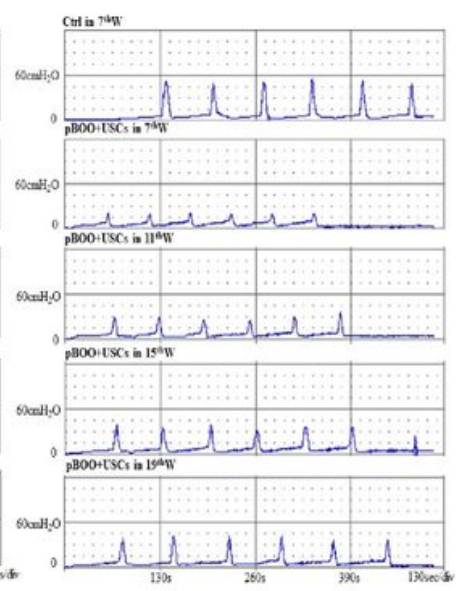

\section{Figure 1}

(A-F) Cystometric parameters, end-filling pressure, residual volume, and bladder capacity were increased and maximal voiding pressure, voided volume, and bladder compliance were decreased in pBOO rats. Injection with USCs improved bladder micturition function with respect to end-filling pressure, maximal voiding pressure, and voided volume, thereby finally increasing the bladder compliance. a: $p<0.05$, vs. control group, b: $p>0.05$, vs. pBOO group, c: $p<0.05$, vs. pBO0 group, $d: p>0.05$, vs. control group. (G) Representative cystometrograms of three rat groups $7,11,15$, and 19 weeks after urethral ligation. 
A

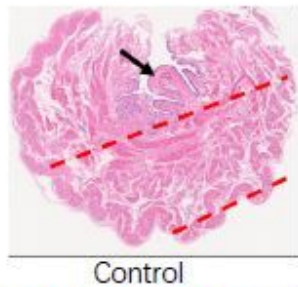

B

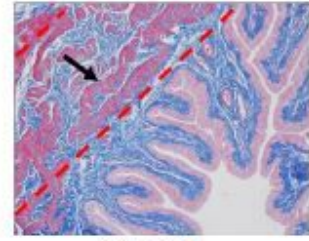

Control

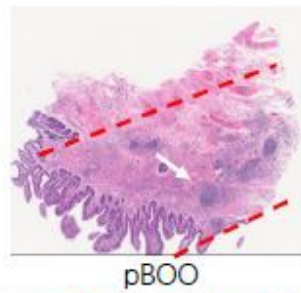

$\mathrm{pBOO}$

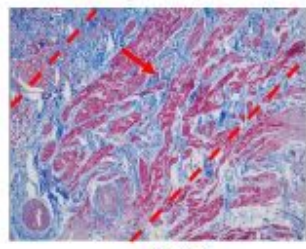

$\mathrm{pBOO}$

D

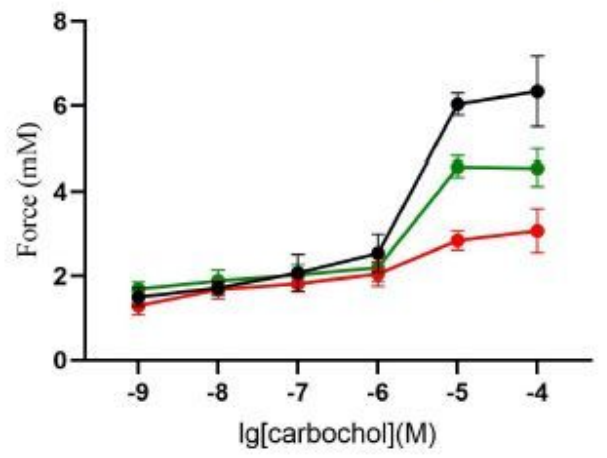

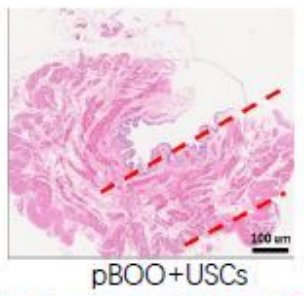

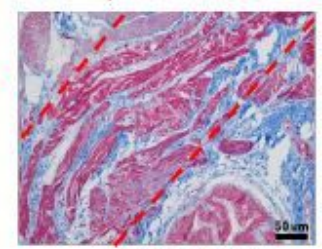

$\mathrm{pBOO}+$ USCs

$\rightarrow$ Ctrl

$\rightarrow$ pBOO

$\rightarrow$ pBOO+USCs
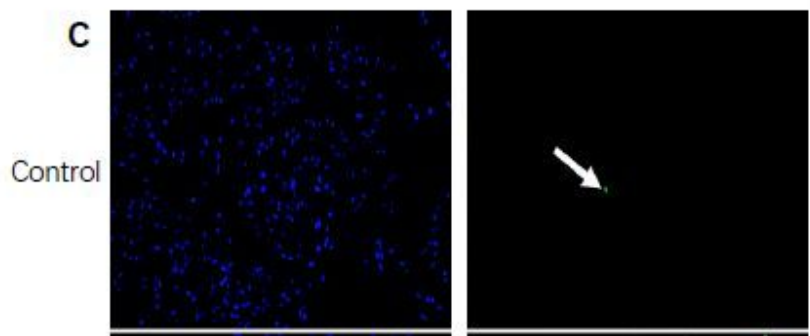

$\mathrm{pBOO}$
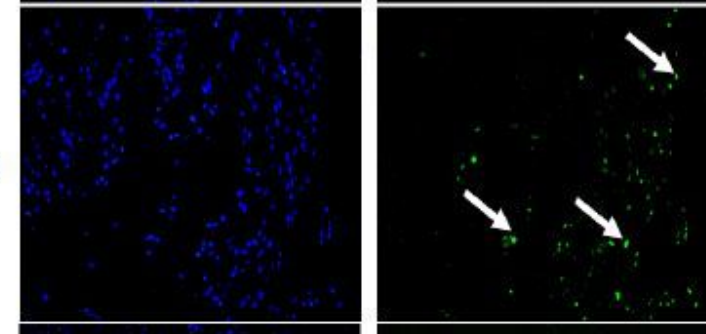

pBOO+

USCS

50 um

Figure 2

(A-C) Hematoxylin and eosin staining, Masson's trichrome staining, and TUNEL assay revealed the deposition of a fibrous matrix and cellular apoptosis in the bladder wall, and USC treatment improved the fibrosis and cell apoptosis in the bladder wall of pBOO+USCs rats. (D) The detrusor contractility, induced by carbachol, was significantly lower in pBOO rats than in control rats. The pBOO+USCs rats had a higher sensitivity to carbachol in detrusor contraction. 

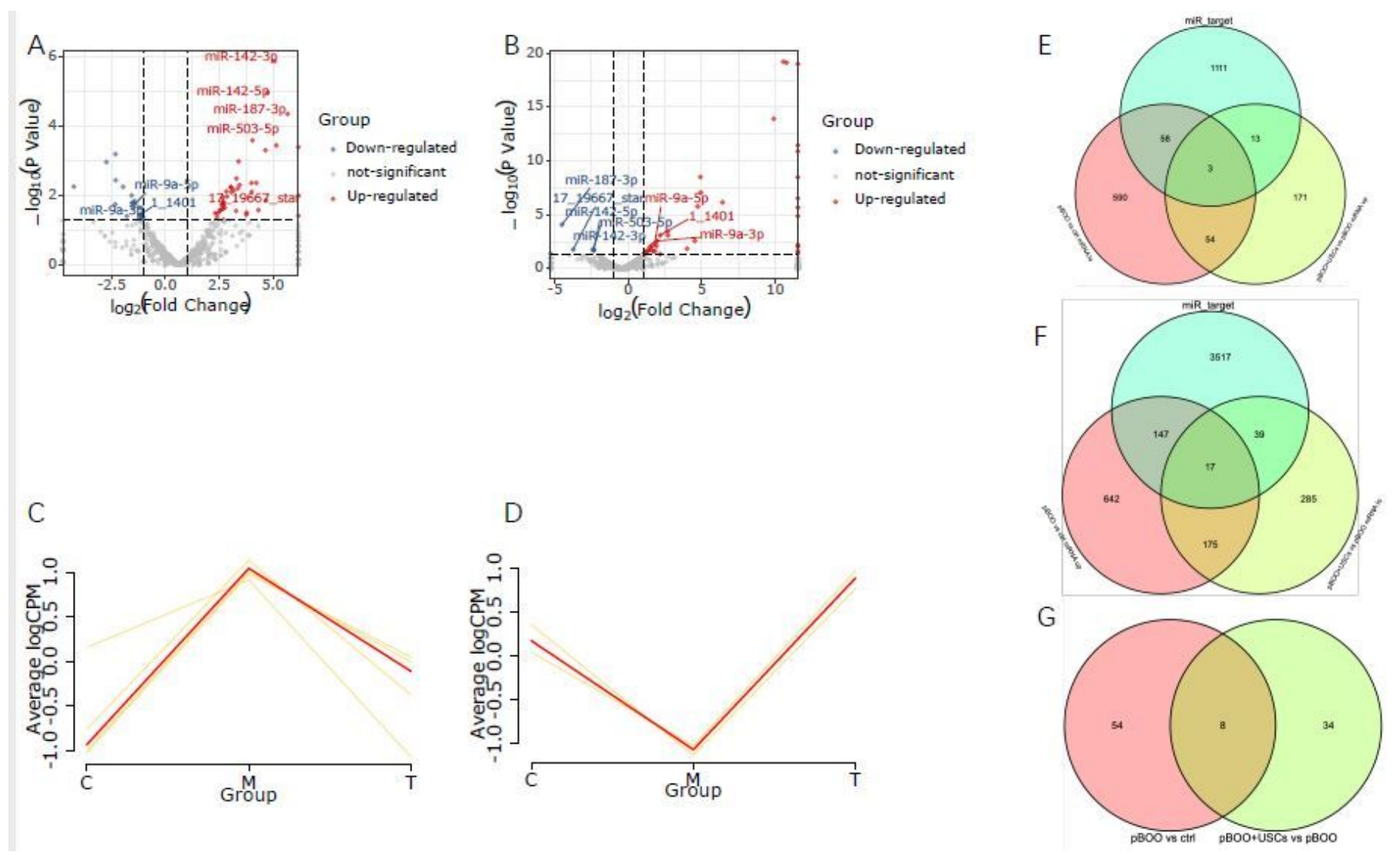

Figure 3

Volcano plots of differentially expressed miRNAs (DEMs) in rats in the pBOO vs. control group $(A)$ and the pBO0+USCs vs. pBOO group (B). Log2 transformation of the fold change in miRNA expression between each group is plotted on the $x$-axis. The log $p$ value (base 10) is plotted on the $y$-axis. DEMs (fold change $\geq 1$ ) are indicated in red (upregulated miRNAs) and blue (downregulated miRNAs). (C and D) The gene trend of DEMs in the three groups. (E) The overlapping target genes of the five DEMs and downregulated mRNAs in rats from pBOO vs. control group (pBOO vs. ctrl mRNA_lo) and upregulated mRNAs in rats from $\mathrm{pBOO+USCs}$ vs. $\mathrm{pBOO}$ group ( $\mathrm{pBOO+USCs}$ vs. pBOO mRNA_up) were identified using Venn diagrams. $(F)$ The overlapping target genes of the five DEMs and upregulated mRNAs in rats from $\mathrm{pBOO}$ vs. control group ( $\mathrm{pBOO}$ vs. ctrl mRNA_up) and downregulated mRNAs in rats from pBOO+USCs vs. pBOO group (pBOO+USCs vs. pBOO mRNA_lo) were identified using Venn diagrams. 


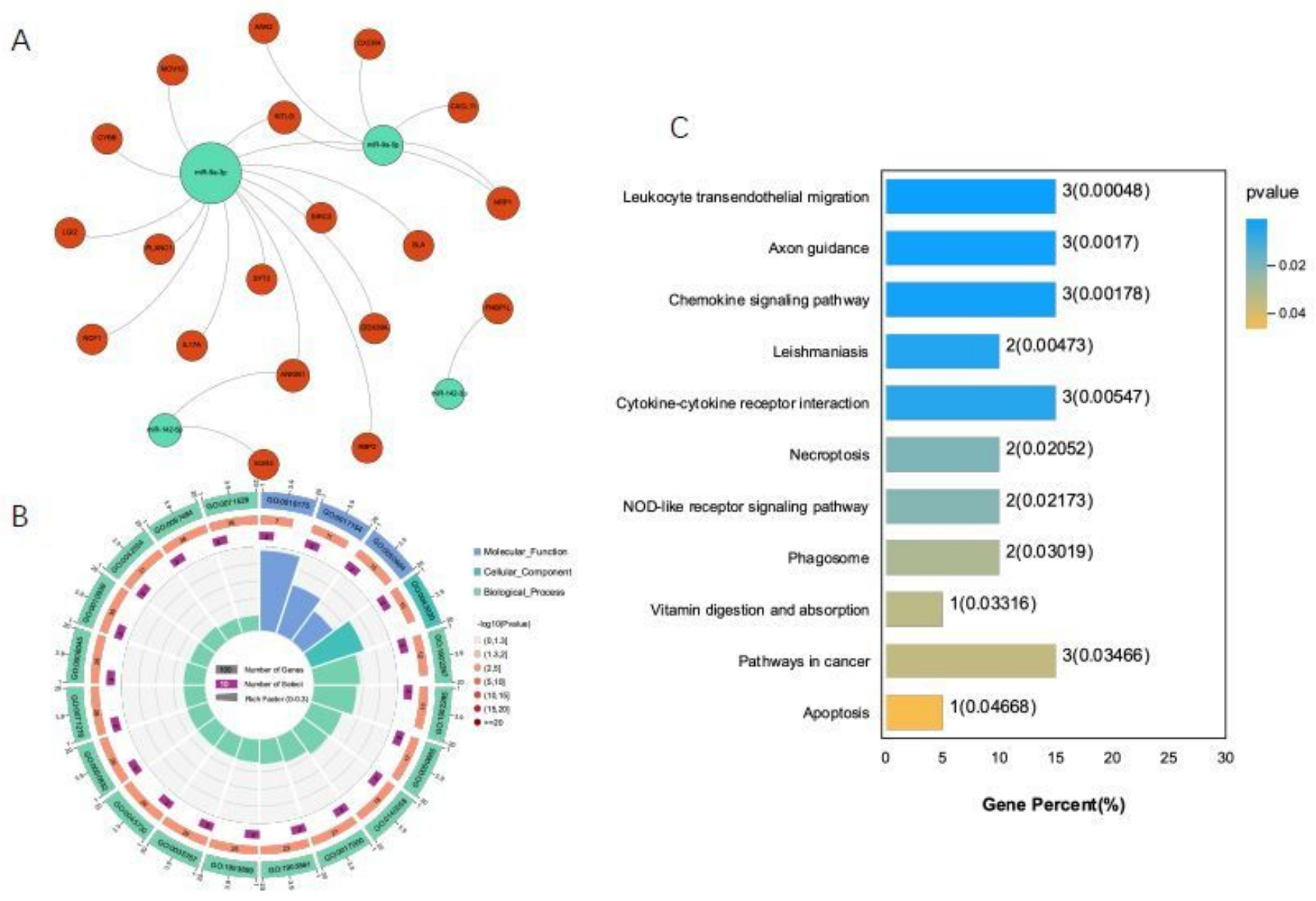

Figure 4

(A) Regulation networks of miRNA-mRNA were constructed using the Gephi software (version 0.9.1). Green dots represent miRNAs, whereas red dots indicate downstream target genes. (B) Enriched GO terms for target genes. (C) KEGG analysis of the genes in the regulation networks of miRNA-mRNA. 